\title{
Sparse polynomial exponential sums
}

\author{
by \\ Todd Cochrane, Christopher Pinner and \\ Jason Rosenhouse (Manhattan, KS)
}

1. Introduction. In this paper we estimate the complete exponential sum

$$
S(f, q)=\sum_{x=1}^{q} e_{q}(f(x)),
$$

where $e_{q}(\cdot)$ is the additive character $e_{q}(\cdot)=e^{2 \pi i \cdot / q}$, and $f$ is a sparse integer polynomial,

$$
f(x)=a_{1} x^{k_{1}}+\ldots+a_{r} x^{k_{r}}
$$

with $0<k_{1}<\ldots<k_{r}$. We always assume that the content of $f,\left(a_{1}, \ldots, a_{r}\right)$, is relatively prime to the modulus $q$. Let $d=d(f)=k_{r}$ denote the degree of $f$ and for any prime $p$ let $d_{p}(f)$ denote the degree of $f$ read modulo $p$. A fundamental problem is to determine whether there exists an absolute constant $C$ such that for an arbitrary positive integer $q$,

$$
|S(f, q)| \leq C q^{1-1 / d}
$$

if $f$ is not a constant function modulo $p$ for each prime $p \mid q$. It is well known that the exponent $1-1 / d$ is best possible. For the case of Gauss sums $(r=1)$ Shparlinski [26], [27] showed that one may take $C=1+O\left(d^{-1 / 4+\varepsilon}\right)$ and this was sharpened to $C=1+O\left(d^{-1+\varepsilon}\right)$ in his subsequent work with Konyagin [14, Theorem 6.7].

The best upper bounds available for general $f$ are

$$
|S(f, q)| \leq e^{d+O(d / \log d)} q^{1-1 / d},
$$

due to Stechkin [29], and

$$
|S(f, q)| \leq e^{1.74 d} q^{1-1 / d},
$$

2000 Mathematics Subject Classification: 11L07, 11L03.

Key words and phrases: exponential sums.

The research of the second author was supported in part by the National Science Foundation under grant EPS-9874732 and matching support from the State of Kansas. 
due to Qi and Ding [25]; see also Chen [2], [3], Hua [11]-[13], Lu [17]-[19], Nechaev [20], [21], Nechaev and Topunov [22], Qi and Ding [23], [24] and Zhang and Hong [31]. These authors noted that in order to make any further improvement one must first obtain a nontrivial upper bound on the prime modulus exponential sum $|S(f, p)|$ for $p<(d-1)^{2}$, the interval where Weil's [30] bound $|S(f, p)| \leq(d-1) \sqrt{p}$ is worse than the trivial bound. In [5] we obtained a bound of this type in terms of the number of terms $r$ of $f(x)$. Using this bound we establish here

TheOREM 1.1. For any positive integer $r$ there exists a constant $C(r)$ such that for any polynomial $f$ of type (1.2) and positive integer $q$ relatively prime to the content of $f$,

$$
|S(f, q)| \leq C(r) q^{1-1 / d} .
$$

Although our proof yields $C(r) \leq e^{O\left(r^{4}\right)}$, no attempt was made to obtain the best possible value for $C(r)$.

For prime power moduli one can replace $C(r)$ with an absolute constant as shown by Stechkin [29] and Cochrane and Zheng [8], the latter result being

Lemma 1.1 [8, Theorem 1.1]. Let $f$ be a polynomial over $\mathbb{Z}$ of degree $d$ and $p$ a prime with $d_{p}(f) \geq 1$. Then for any $m \geq 1$,

$$
\left|S\left(f, p^{m}\right)\right| \leq 4.41 p^{m(1-1 / d)} .
$$

It is also well known (see [20], [3] or [8]) that for $p \geq(d-1)^{2 d /(d-2)}$ and $m \geq 1$,

$$
\left|S\left(f, p^{m}\right)\right| \leq p^{m(1-1 / d)} .
$$

The significance of the constant one in (1.5) lies in the fact that bounds for exponential sums modulo prime powers lead to bounds for a general modulus $q=\prod_{i=1}^{k} p_{i}^{e_{i}}$ via the multiplicative formula

$$
S(f, q)=\prod_{i=1}^{k} S\left(\lambda_{i} f, p_{i}^{e_{i}}\right),
$$

where the $\lambda_{i}$ are such that $\sum_{i=1}^{k} \lambda_{i} q / p_{i}^{e_{i}}=1$. Thus if (1.5) holds for all prime power divisors of $q$ then it follows that $|S(f, q)| \leq q^{1-1 / d}$. It is desirable to extend the inequality in (1.5) to an interval of the type $p>C d$ for some constant $C$.

In closing we note that for sums over reduced residue systems,

$$
S^{*}(f, q)=\sum_{x=1,(x, q)=1}^{q} e_{q}(f(x)),
$$


the exponent in the upper bound can be dramatically reduced. Shparlinski [28] showed that

$$
\left|S^{*}(f, q)\right| \leq C(d, \varepsilon) q^{1-1 / r+\varepsilon}
$$

for any sparse polynomial in $r$ terms with content relatively prime to $q$. Loh [16] obtained a related upper bound but an error in his Lemma 3 leaves his results in doubt.

2. The method of recursion. A standard method for bounding exponential sums modulo prime powers is the method of recursion, also known as the method of critical points. For any polynomial $f$ let $t=t_{p}(f)=$ $\operatorname{ord}_{p}\left(f^{\prime}\right)$ be the largest power of $p$ dividing all of the coefficients of $f^{\prime}$, $d_{1}=d_{p}\left(p^{-t} f^{\prime}\right)$, and let $\mathcal{A}=\mathcal{A}(f, p)$ be the set of zeros of the congruence $p^{-t} f^{\prime}(x) \equiv 0(\bmod p)$. $\mathcal{A}$ is called the set of critical points associated with the sum $S\left(f, p^{m}\right)$, for any $m \geq 2$. Write

$$
S\left(f, p^{m}\right)=\sum_{\alpha=0}^{p-1} S_{\alpha}\left(f, p^{m}\right)
$$

with

$$
S_{\alpha}\left(f, p^{m}\right)=\sum_{x \equiv \alpha(\bmod p)} e_{p^{m}}(f(x)) .
$$

A fact of central importance is that if $m$ is sufficiently large then $S_{\alpha}\left(f, p^{m}\right)$ $=0$ unless $\alpha$ is a critical point.

Lemma 2.1 [6, Proposition 4.1]. Suppose that $p$ is an odd prime and $m \geq t+2$, or $p=2$ and $m \geq t+3$, or $p=2, t=0$ and $m=2$. Then if $\alpha$ is not a critical point, $S_{\alpha}\left(f, p^{m}\right)=0$. Consequently,

$$
S\left(f, p^{m}\right)=\sum_{\alpha \in \mathcal{A}} S_{\alpha}\left(f, p^{m}\right)
$$

For any $\alpha \in \mathcal{A}$ define

$$
\begin{aligned}
& \sigma=\sigma_{\alpha}:=\operatorname{ord}_{p}(f(p x+\alpha)-f(\alpha)), \\
& g_{\alpha}(x):=p^{-\sigma}(f(p x+\alpha)-f(\alpha)) .
\end{aligned}
$$

LEMma 2.2 [6, Proposition 4.1] (The recursion relationship). Suppose that $p$ is an odd prime and $m \geq t+2$, or $p=2$ and $m \geq t+3$, or $p=2$, $t=0$ and $m=2$. Then if $\alpha \in \mathcal{A}$,

$$
S_{\alpha}\left(f, p^{m}\right)=e_{p^{m}}(f(\alpha)) p^{\sigma-1} S\left(g_{\alpha}, p^{m-\sigma}\right),
$$

where

$$
S\left(g_{\alpha}, p^{m-\sigma}\right)= \begin{cases}\sum_{x=1}^{p^{m-\sigma}} e_{p^{m-\sigma}}\left(g_{\alpha}(x)\right) & \text { if } m>\sigma \\ p^{m-\sigma} & \text { if } m \leq \sigma\end{cases}
$$


Under the hypotheses of the lemma we have

$$
\left|S\left(f, p^{m}\right)\right| \leq \sum_{\alpha \in \mathcal{A}}\left|S_{\alpha}\left(f, p^{m}\right)\right|=\sum_{\alpha \in \mathcal{A}} p^{\sigma_{\alpha}-1}\left|S\left(g_{\alpha}, p^{m-\sigma_{\alpha}}\right)\right| .
$$

In particular, since there are at most $d_{1}$ critical points we immediately have the upper bound

$$
\left|S\left(f, p^{m}\right)\right| \leq d_{1} p^{m-1} .
$$

In [8] we established the following bounds for $S_{\alpha}\left(f, p^{m}\right)$ and $S\left(f, p^{m}\right)$ :

Lemma 2.3 [8, Theorem 2.1]. Let $f$ be a polynomial over $\mathbb{Z}$ and $p$ a prime with $d_{p}(f) \geq 1$. Suppose that $p$ is odd and $m \geq t+2$, or $p=2$ and $m \geq t+3$. Set $\lambda=(5 / 4)^{5} \approx 3.05$ and $d_{1}=d_{p}\left(p^{-t} f^{\prime}\right)$. Then

(i) For any critical point $\alpha$ of multiplicity $\nu$ we have

$$
\left|S_{\alpha}\left(f, p^{m}\right)\right| \leq \min \{\nu, \lambda\} p^{t /(\nu+1)} p^{m(1-1 /(\nu+1))},
$$

with equality if $\nu=1$.

(ii) $\left|S\left(f, p^{m}\right)\right| \leq \lambda p^{t /\left(d_{1}+1\right)} p^{m\left(1-1 /\left(d_{1}+1\right)\right)}$.

Related results using the method of critical points were obtained by Chalk [1], Cochrane [4], Cochrane and Zheng [6], [7], Ding [9], [10], and Loh [15].

For any critical point $\alpha$ set

$$
\tau:=\operatorname{ord}_{p}\left(g_{\alpha}^{\prime}(x)\right), \quad g_{1}(x):=p^{-\tau} g_{\alpha}^{\prime}(x) .
$$

The following relations are well known (see e.g. [6, Lemma 3.1]) and play a central role in the proof of the preceding lemma.

LEMMA 2.4.

$$
\begin{aligned}
& \sigma \geq\left\{\begin{array}{l}
t+2 \quad \text { if } p \text { is odd or } \nu>1 \\
t+1 \quad \text { if } p=2 \text { and } \nu=1
\end{array}\right. \\
& \sigma \leq \nu+1+t-\tau . \\
& d_{p}\left(g_{\alpha}\right) \leq\left\{\begin{array}{l}
\sigma-t+\operatorname{ord}_{p}\left(d_{p}\left(g_{\alpha}\right)\right) \leq \nu+1+\operatorname{ord}_{p}\left(d_{p}\left(g_{\alpha}\right)\right) \\
\sigma \leq \nu+1+t-\tau .
\end{array}\right. \\
& d_{p}\left(g_{1}\right) \leq \sigma+\tau-t-1 \leq \nu \\
& p^{\tau} \mid d_{p}\left(g_{\alpha}\right)
\end{aligned}
$$

An immediate consequence that we frequently make reference to is

Lemma 2.5. Suppose that $\alpha$ is a critical point of multiplicity $\nu$ with $\nu \geq 2$ and $p>\nu+2$. Then $d_{p}\left(g_{\alpha}\right) \leq \nu+1$.

Proof. Let $d_{p}=d_{p}\left(g_{\alpha}\right)$. Suppose that $\operatorname{ord}_{p}\left(d_{p}\right) \geq 1$. If $d_{p}=p$ then by (2.10) we have $p=d_{p} \leq \nu+2$ contradicting our assumption. Otherwise $d_{p} \geq 2 p$ and we have $p \leq d_{p} / 2 \leq d_{p}-\operatorname{ord}_{p}\left(d_{p}\right) \leq \nu+1$, again a contradiction. Thus $p \nmid d_{p}$ and we obtain (by (2.10)) $d_{p} \leq \nu+1$. 
3. Preliminary upper bounds. We begin with a couple of auxiliary lemmas.

LEMMA 3.1. Define $\lambda_{i}=i$ for $i=1,2,3$ and $\lambda_{i}=\lambda$ for $i \geq 4$, where $\lambda=(5 / 4)^{5} \approx 3.05$. Then for $1 \leq i \leq d$ we have

$$
d \lambda_{i} \lambda^{(i-d) /(i+1)} \leq i \lambda .
$$

Proof. For any fixed $i \geq 1$ the function $f_{i}(x):=\left(\lambda_{i} / i\right) x \lambda^{(i-x) /(i+1)}$ attains its maximum value at $x=(i+1) / \log (\lambda)<i+1$, and is decreasing for larger values of $x$. Thus for $d \geq i$, the maximum value of $f_{i}(d)$ occurs at $d=i$ or $d=i+1$. Now, $f_{i}(i)=\lambda_{i} \leq \lambda$ and $f_{i}(i+1)=\lambda_{i}(1+1 / i) \lambda^{-1 /(i+1)} \leq \lambda$, as can be seen by considering the different cases $i=1,2,3$ and $i \geq 4$.

LEMMA 3.2. If $p>c d_{1}$ for some constant $c$ then for $1 \leq i \leq d_{1}-1$ we have

$$
\left(4 p /\left(c d_{1}\right)\right)^{\left(i-d_{1}\right) /(i+1)} \leq i / d_{1} .
$$

Proof. We first note that

$$
\left(d_{1} / i\right)^{(i+1) /\left(d_{1}-i\right)} \leq 4 \quad \text { for } 1 \leq i \leq d_{1}-1 .
$$

This can be checked directly for $i=1,2,3$. For $i \geq 4$ it follows from Lemma 3.1. Then $p>c d_{1} \geq(c / 4) d_{1}\left(d_{1} / i\right)^{(i+1) /\left(d_{1}-i\right)}$ and the result follows.

LEMMA 3.3. Let $p$ be a prime and $f$ be any integer polynomial with $t=0$ and either $d_{1}=0,1$ or $p>d_{1}^{2+4 /\left(d_{1}-1\right)}$ where $d_{1}=d_{p}\left(p^{-t} f^{\prime}\right)$. Then for $m \geq 2$,

$$
\left|S\left(f, p^{m}\right)\right| \leq p^{m\left(1-1 /\left(d_{1}+1\right)\right)} .
$$

Proof. If $d_{1}=0$ then there are no critical points and the sum is zero. If $d_{1}=1$ then there is a single critical point of multiplicity one and the result follows from Lemma 2.3(i). Suppose that $d_{1} \geq 2$. Let $\mathcal{A}=\mathcal{A}(f, p) \subset \mathbb{F}_{p}$ be the set of critical points. We prove by induction on $m$ that, under the hypotheses of the theorem,

$$
\left|S_{\alpha}\right| \leq p^{m(1-1 /(\nu+1))}
$$

for any critical point $\alpha \in \mathcal{A}$. We first note that (3.1) is an immediate consequence of $(3.2)$. Indeed, if $p^{m} \leq\left(p / d_{1}\right)^{d_{1}+1}$ then using the trivial upper bound $\left|S_{\alpha}\left(f, p^{m}\right)\right| \leq p^{m-1}$ we have $\left|S\left(f, p^{m}\right)\right| \leq \sum_{\alpha \in \mathcal{A}}\left|S_{\alpha}\left(f, p^{m}\right)\right| \leq$ $d_{1} p^{m-1} \leq p^{m\left(1-1 /\left(d_{1}+1\right)\right)}$. Next, if there is a critical point $\alpha$ of multiplicity $d_{1}$ then it is the only critical point and we have $\left|S\left(f, p^{m}\right)\right|=\left|S_{\alpha}\left(f, p^{m}\right)\right| \leq$ $p^{m\left(1-1 /\left(d_{1}+1\right)\right)}$.

Finally, suppose that $p^{m}>\left(p / d_{1}\right)^{d_{1}+1}$ and that every critical point is of multiplicity less than $d_{1}$. Letting $n_{i}$ denote the number of critical points of 
multiplicity $i$ we deduce from (3.2) that

$$
\begin{aligned}
\left|S\left(f, p^{m}\right)\right| & \leq \sum_{\alpha \in \mathcal{A}}\left|S_{\alpha}\left(f, p^{m}\right)\right| \leq \sum_{i=1}^{d_{1}-1} n_{i} p^{m(1-1 /(i+1))} \\
& =p^{m\left(1-1 /\left(d_{1}+1\right)\right)}\left(\sum_{i=1}^{d_{1}-1} n_{i} p^{m\left(i-d_{1}\right) /\left((i+1)\left(d_{1}+1\right)\right)}\right) .
\end{aligned}
$$

Then from $p^{m}>\left(p / d_{1}\right)^{d_{1}+1}, p>4 d_{1}$ and Lemma 3.2 with $c=4$ we obtain

$$
\begin{aligned}
\left|S\left(f, p^{m}\right)\right| & \leq p^{m\left(1-1 /\left(d_{1}+1\right)\right)}\left(\sum_{i=1}^{d_{1}-1} n_{i}\left(p / d_{1}\right)^{\left(i-d_{1}\right) /(i+1)}\right) \\
& \leq\left(\sum_{i=1}^{d_{1}-1} n_{i} i / d_{1}\right) p^{m\left(1-1 /\left(d_{1}+1\right)\right)} \leq p^{m\left(1-1 /\left(d_{1}+1\right)\right)} .
\end{aligned}
$$

We now proceed to establish (3.2). If $\nu=1$ then by Lemma 2.3 we have equality in (3.2). So we may assume that $\nu \geq 2$. When $m=2$ the bound is trivial, $\left|S_{\alpha}\right| \leq p \leq p^{2(1-1 /(\nu+1))}$. Suppose $m \geq 3$. If $\sigma \geq m$ then the result follows trivially,

$$
\left|S_{\alpha}\right| \leq p^{m-1} \leq p^{m(1-1 /(\nu+1))} p^{(\sigma-\nu-1) /(\nu+1)} \leq p^{m(1-1 /(\nu+1))},
$$

the latter inequality following from (2.9). Suppose next that $\sigma=m-1$. Put $d_{p}=d_{p}\left(g_{\alpha}\right)$. Since $p>d_{1}^{2} \geq \nu^{2} \geq \nu+2$ it follows from Lemma 2.5 that $d_{p} \leq \nu+1 \leq d_{1}+1$. If $d_{p} \geq 3$ then $p \geq\left(d_{p}-1\right)^{2+4 /\left(d_{p}-2\right)}$, so by the Weil bound, $\left|S\left(g_{\alpha}, p\right)\right| \leq\left(d_{p}-1\right) \sqrt{p} \leq p^{1-1 / d_{p}} \leq p^{1-1 /(\nu+1)}$. If $d_{p}=1$ or 2 the same bound is elementary. It follows from the recursion formula of Lemma 2.2 that

$$
\begin{aligned}
\left|S_{\alpha}\right| & =p^{\sigma-1}\left|S\left(g_{\alpha}, p\right)\right| \\
& \leq p^{m-1-1 /(\nu+1)}=p^{(\sigma-\nu-1) /(\nu+1)} p^{m(1-1 /(\nu+1))} \leq p^{m(1-1 /(\nu+1))} .
\end{aligned}
$$

Suppose finally that $m \geq \sigma+2$. We note that $\tau=0$ since by $(2.12), p^{\tau} \leq$ $d_{p}\left(g_{\alpha}\right) \leq \nu+1 \leq d_{1}+1<p$, and so we can apply the induction assumption to $S\left(g_{\alpha}, p^{m-\sigma}\right)$. Putting $d_{2}=d_{p}\left(g_{\alpha}^{\prime}\right) \leq \nu \leq d_{1}$ and noting that either $d_{2}=0,1$ or $p \geq d_{2}^{2+2 /\left(d_{2}-1\right)}$ we obtain

$$
\begin{aligned}
\left|S_{\alpha}\right| & =p^{\sigma-1}\left|S\left(g_{\alpha}, p^{m-\sigma}\right)\right| \leq p^{\sigma-1} p^{(m-\sigma)\left(1-1 /\left(d_{2}+1\right)\right)} \\
& \leq p^{\sigma-1} p^{(m-\sigma)(1-1 /(\nu+1))} \leq p^{m(1-1 /(\nu+1))}
\end{aligned}
$$

4. Multiplicity estimates. Next, we obtain an upper bound on the multiplicity of a nonzero zero of a sparse polynomial

$$
f(x)=a_{1} x^{k_{1}}+\ldots+a_{r} x^{k_{r}}(\bmod p) .
$$


Let $a \not \equiv 0(\bmod p)$ be a zero of multiplicity $\nu(\bmod p)$, that is,

$$
(x-a)^{\nu} \| f(x)(\bmod p) .
$$

For $1 \leq i \leq r$ let

$$
S(i, \alpha)=\left\{k_{j}: k_{j} \equiv k_{i}\left(\bmod p^{\alpha}\right)\right\}
$$

and set

$$
\begin{aligned}
\alpha_{i} & =\max \{\alpha:|S(i, \alpha)| \geq 2\}, \\
r_{i} & =\left|S\left(i, \alpha_{i}\right)\right| .
\end{aligned}
$$

LEMMA 4.1. The multiplicity $\nu$ of any nonzero zero of $f(x)(\bmod p)$ satisfies $\nu<\min _{i} r_{i} p^{\alpha_{i}}$. In particular, if $p$ does not divide any $k_{i}-k_{j}$ with $i \neq j$ then $\nu<r$.

Lemma 4.1 follows from the more precise

LEMMA 4.2. Suppose that $k_{1}, \ldots, k_{t}$ are the smallest distinct exponents modulo $p$ so that

$$
f(x)=x^{k_{1}} f_{1}(x)^{p}+\ldots+x^{k_{t}} f_{t}(x)^{p}(\bmod p)
$$

where

$$
f_{i}(x)=\sum_{k_{j}=k_{i}+l_{j} p} a_{j} x^{l_{j}} .
$$

Then if $f(x)$ has a nonzero zero a of multiplicity $\nu(\bmod p)$, we have

$$
\nu=k p+u
$$

where $u<t$ and $(x-a)^{k}$ is the highest power dividing all the $f_{1}, \ldots, f_{t}$.

Proof. Suppose that $(x-a)^{k} \mid f_{1}, \ldots, f_{t}$ with $(x-a)^{k+1} \nmid f_{1}$, and write $f_{i}(x)=(x-a)^{k} g_{i}(x)(\bmod p), \nu=k p+u$, so that

$$
(x-a)^{u} \| g(x)=x^{k_{1}} g_{1}(x)^{p}+\ldots+x^{k_{t}} g_{t}(x)^{p} \text {. }
$$

Writing $\nabla=x \frac{d}{d x}$ we must have $\nabla^{i} g(a) \equiv 0(\bmod p)$ for $j=0, \ldots, u-1$. That is,

$$
k_{1}^{j} a^{k_{1}} g_{1}(a)^{p}+\ldots+k_{t}^{j} a^{k_{t}} g_{t}(a)^{p} \equiv 0(\bmod p)
$$

for $j=0, \ldots, u-1$. Since $\left|\operatorname{det}\left(k_{i}^{j}\right)_{i=1, \ldots, t, j=0, \ldots, t-1}\right|=\prod\left|k_{i}-k_{j}\right| \not \equiv 0(\bmod p)$ and $a^{k_{1}} g_{1}(a)^{p} \not \equiv 0(\bmod p)$ we must therefore have $u<t$.

Proof of Lemma 4.1. Pick an arbitrary $k_{i}, i=1, \ldots, t$, and use the preceding lemma and induction on $\alpha_{i}$ : If $\alpha_{i}=0$ then plainly $k=0$ and $\nu=u<t \leq r=r_{i}$. If $\alpha_{i} \geq 1$ then since $(x-a)^{k} \mid f_{i}(x)$ we have (by induction) $k<r_{i} p^{\alpha_{i}-1}$ and $u<p$ giving

$$
\nu=p k+u \leq\left(r_{i} p^{\alpha_{i}-1}-1\right) p+(p-1)<r_{i} p^{\alpha_{i}} .
$$

In practice we apply the multiplicity estimate to the polynomial $p^{-t} f^{\prime}(x)$ and so we let $r_{1}=r_{1}(f, p)$ be the number of nonzero terms modulo $p$ of the polynomial $p^{-t} f^{\prime}(x)$. For critical points having multiplicity less than $r_{1}$ we have the following upper bound. 
LEMMA 4.3. Let $f$ be a sparse polynomial as in (1.2) and suppose that either $r_{1}=1,2$ or $p>\left(r_{1}-1\right)^{2 r_{1} /\left(r_{1}-2\right)}$. Then if $m \geq t+2$ and $\alpha$ is a critical point of multiplicity $\nu<r_{1}$ we have

$$
\left|S_{\alpha}\right| \leq p^{t /(\nu+1)} p^{m(1-1 /(\nu+1))} .
$$

Proof. If $\nu=1$ the result follows from Lemma 2.3, and so we may assume $\nu \geq 2$ and $r_{1} \geq 3$. Let $d_{p}=d_{p}\left(g_{\alpha}\right)$. Since $p>\nu^{2+4 /(\nu-1)}$, we get $d_{p} \leq \nu+1$ by Lemma 2.5 , and thus $p>\left(d_{p}-1\right)^{2 d_{p} /\left(d_{p}-2\right)}$. Also, since $p^{\tau} \leq d_{p}\left(g_{\alpha}\right) \leq \nu+1 \leq r_{1}+1<p$ we must have $\tau=0$.

If $\sigma \geq m$ the result follows trivially,

$$
\left|S_{\alpha}\right| \leq p^{m-1}=p^{(m-\nu-1) /(\nu+1)} p^{m(1-1 /(\nu+1))} \leq p^{t /(\nu+1)} p^{m(1-1 /(\nu+1))} .
$$

Suppose next that $\sigma=m-1$. Then applying the bound in (1.5) to $S\left(g_{\alpha}, p\right)$, we obtain

$$
\begin{aligned}
\left|S_{\alpha}\right| & =p^{\sigma-1}\left|S\left(g_{\alpha}, p\right)\right| \leq p^{\sigma-1} p^{1-1 /(\nu+1)} \\
& =p^{(\sigma-\nu-1) /(\nu+1)} p^{m(1-1 /(\nu+1))} \leq p^{t /(\nu+1)} p^{m(1-1 /(\nu+1))} .
\end{aligned}
$$

Finally, if $\sigma \leq m-2$ then we can apply Lemma 3.3 to $S\left(g_{\alpha}, p^{m-\sigma}\right)$, since $d_{2}:=d_{p}\left(g_{\alpha}^{\prime}\right) \leq \nu<r_{1}$ and so $p \geq d_{2}^{2+4 /\left(d_{2}-1\right)}$. We obtain

$$
\begin{aligned}
\left|S_{\alpha}\right| & \leq p^{\sigma-1}\left|S\left(g_{\alpha}, p^{m-\sigma}\right)\right| \\
& \leq p^{\sigma-1} p^{(m-\sigma)\left(1-1 /\left(d_{2}+1\right)\right)}=p^{(\sigma-\nu-1) /(\nu+1)} p^{m(1-1 /(\nu+1))} .
\end{aligned}
$$

5. Bounds for exponential sums with $p$ small relative to $d$. First we consider sums modulo $p$. From the bound of Weil, one deduces (see [8, Lemma 3.1]) the upper bound

$$
|S(f, p)| \leq 1.75 p^{1-1 / d}
$$

for any polynomial $f$ with $d_{p}(f) \geq 1$. Moreover the constant 1.75 may be replaced by 1 provided $p \gg d^{2}$. For our purposes here we need the constant 1 for $p \gg d$. We obtain this from the following result established in the authors' work [5, Corollary 1.1].

LEMMA 5.1. Let $f$ be an integer polynomial of degree $d$ as in (1.2). Then for any $\delta>0$, if $p>\left(9 / \delta^{1.06}\right) d$ and $p>C_{1}(\delta)$, then

$$
\left|\sum_{x=1}^{p} e_{p}(f(x))\right| \leq p\left(1-\frac{1}{r p^{\delta}}\right) .
$$

Lemma 5.2. Let $f$ be a polynomial as in (1.2) of degree $d=d_{p}(f) \geq 1$ $(\bmod p)$ and suppose that $p>C_{2}$ (an absolute constant), $p>50 d$ and $p>r^{4}$. Then

$$
|S(f, p)| \leq p^{1-1 / d} .
$$

Proof. The result is elementary for $d=1,2$ and so we assume $d>2$. If $p>16 d^{2}$ then the result follows from the Weil bound $|S(f, p)| \leq(d-1) \sqrt{p}$. 
Suppose that $p \leq 16 d^{2}$. Applying Lemma 5.1 with $\delta=1 / 5$ we deduce that if $p>50 d$ and $p>C_{1}(1 / 5)$ then $|S(f, p)| \leq p\left(1-1 /\left(r p^{1 / 5}\right)\right)$. Since $p>r^{4}$ it follows that $|S(f, p)| \leq p\left(1-1 / p^{9 / 20}\right)$, and since $p \leq 16 d^{2}$ the latter is $\leq p^{1-1 / d}$ for $p>10^{60}$.

Lemma 5.3. Let $f$ be a sparse polynomial as in (1.2) with $p \geq 50\left(d_{1}+1\right)$, $p>C_{2}$ (the constant in Lemma 5.2), $p>r^{4}$ and $m \geq t+2$. Then for any critical point $\alpha$ of multiplicity $\nu$ we have

$$
\left|S_{\alpha}\right| \leq p^{t /(\nu+1)} p^{m(1-1 /(\nu+1))}
$$

and

$$
\left|S\left(f, p^{m}\right)\right| \leq p^{t /\left(d_{1}+1\right)} p^{m\left(1-1 /\left(d_{1}+1\right)\right)} .
$$

Proof. We first observe that (5.4) is always an immediate consequence of (5.3). Indeed, if $p^{m-t} \leq\left(p / d_{1}\right)^{d_{1}+1}$ then using the trivial upper bound $\left|S_{\alpha}\left(f, p^{m}\right)\right| \leq p^{m-1}$ we have $\left|S\left(f, p^{m}\right)\right| \leq \sum_{\alpha \in \mathcal{A}}\left|S_{\alpha}\left(f, p^{m}\right)\right| \leq d_{1} p^{m-1} \leq$ $p^{t /\left(d_{1}+1\right)} p^{m\left(1-1 /\left(d_{1}+1\right)\right)}$. Next, if there is a critical point $\alpha$ of multiplicity $d_{1}$ then it is the only critical point and we have $\left|S\left(f, p^{m}\right)\right|=\left|S_{\alpha}\left(f, p^{m}\right)\right| \leq$ $p^{t /\left(d_{1}+1\right)} p^{m\left(1-1 /\left(d_{1}+1\right)\right)}$.

Finally, suppose that $p^{m-t}>\left(p / d_{1}\right)^{d_{1}+1}$ and that every critical point is of multiplicity less than $d_{1}$. Letting $n_{i}$ denote the number of critical points of multiplicity $i$ we deduce from (5.3) that

$$
\begin{aligned}
\left|S\left(f, p^{m}\right)\right| & \leq \sum_{i=1}^{d_{1}} n_{i} p^{t /(i+1)} p^{m(1-1 /(i+1))} \\
& \leq p^{t /\left(d_{1}+1\right)} p^{m\left(1-1 /\left(d_{1}+1\right)\right)} \sum_{i} n_{i} p^{(m-t)\left(i-d_{1}\right) /\left((i+1)\left(d_{1}+i\right)\right)} \\
& \leq p^{t /\left(d_{1}+1\right)} p^{m\left(1-1 /\left(d_{1}+1\right)\right)} \sum_{i} n_{i}\left(p / d_{1}\right)^{\left(i-d_{1}\right) /(i+1)} \\
& \leq p^{t /\left(d_{1}+1\right)} p^{m\left(1-1 /\left(d_{1}+1\right)\right)}
\end{aligned}
$$

the last inequality following from Lemma 3.2 (with $c=4$ ) and $\sum_{i} n_{i} i \leq d_{1}$.

We now establish (5.3) by induction on $m$. The result is trivial if $m=2$. Suppose that $m>2$. If $\sigma \geq m$ then from (2.9),

$$
\left|S_{\alpha}\right| \leq p^{m-1} \leq p^{t /(\nu+1)} p^{m(1-1 /(\nu+1))} .
$$

If $\sigma=m-1$ and $\alpha \neq 0$ then since $p>d_{1}$ it follows from Lemma 4.1 that $\nu<r$. Also, since $p \geq 50 d_{1} \geq 50 \nu$ we see by Lemma 2.5 that

$$
d_{p}(g) \leq \nu+1 \leq r<p^{1 / 4},
$$

and so by (1.5), $\left|S\left(g_{\alpha}, p\right)\right| \leq p^{1-1 / d_{p}(g)} \leq p^{1-1 /(\nu+1)}$. It then follows from the recursion relation that

$$
\left|S_{\alpha}\right| \leq p^{\sigma-1}\left|S\left(g_{\alpha}, p\right)\right| \leq p^{\sigma-1 /(\nu+1)} \leq p^{t /(\nu+1)} p^{m(1-1 /(\nu+1))},
$$


by (2.9). If $\alpha=0$ then we have to argue differently since the multiplicity may be larger than $r$. In this case $g_{\alpha}(x)=f(p x)$ is a sparse polynomial with the same number of terms as $f$. Since $p>50\left(d_{1}+1\right) \geq 50(\nu+1) \geq 50 d_{p}\left(g_{\alpha}\right)$ we can apply Lemma 5.2 to obtain $\left|S\left(g_{\alpha}, p\right)\right| \leq p^{1-1 / d_{p}\left(g_{\alpha}\right)}$, and the result follows as before.

Suppose now that $\sigma \leq m-2$. We first note that by (2.12), $\tau=0$ since $p>d_{p}\left(g_{\alpha}\right)$. Set $d_{2}=d_{p}\left(g_{\alpha}^{\prime}\right)$. If $\alpha \neq 0$ then by (2.11) and Lemma 4.1, $d_{2} \leq \nu<r<p^{1 / 4}$. Thus by Lemma 3.3,

$$
\left|S\left(g_{\alpha}, p^{m-\sigma}\right)\right| \leq p^{(m-\sigma)\left(1-1 /\left(d_{2}+1\right)\right)} .
$$

If $\alpha=0$ then we can apply the induction assumption to the polynomial $g_{\alpha}=p^{-\sigma} f(p x)$ and obtain the same bound. From the recursion relationship we then obtain

$$
\begin{aligned}
\left|S\left(f, p^{m}\right)\right| & \leq p^{\sigma-1} p^{(m-\sigma)\left(1-1 /\left(d_{2}+1\right)\right)} \\
& \leq p^{-1+\sigma /(\nu+1)} p^{m(1-1 /(\nu+1))} \leq p^{t /(\nu+1)} p^{m(1-1 /(\nu+1))}
\end{aligned}
$$

Next we obtain a bound valid for even smaller values of $p$. Again, let $d_{1}$ and $r_{1}=r_{1}(f, p)$ be the degree and number of nonzero terms of the polynomial $p^{-t} f^{\prime}(x)$ read modulo $p$.

LEMMA 5.4. Let $f$ be a sparse polynomial in $r$ terms and $p$ a prime with $p>r^{4}, p>C_{3}$ and such that $p \nmid\left(k_{j}-k_{i}\right)$ for all $k_{i}<k_{j} \leq d_{1}$. Then for $m \geq t+2$ and any critical point $\alpha$ of multiplicity $\nu$ we have

(i) If $\alpha \neq 0$ then $\left|S_{\alpha}\left(f, p^{m}\right)\right| \leq p^{t /(\nu+1)} p^{m(1-1 /(\nu+1))}$.

(ii) For $\alpha=0,\left|S_{0}\left(f, p^{m}\right)\right| \leq p^{(2 r+t) /(\nu+1)} p^{m(1-1 /(\nu+1))}$.

(iii) $\left|S\left(f, p^{m}\right)\right| \leq p^{(2 r+t) /\left(d_{1}+1\right)} p^{m\left(1-1 /\left(d_{1}+1\right)\right)}$.

Proof. We take $C_{3}=\max \left\{C_{2}, 200\right\}$ where $C_{2}$ is the constant in Lemma 5.3. The condition $p \nmid\left(k_{i}-k_{j}\right)$ implies (by Lemma 4.1) that $\nu<r_{1}$ for any nonzero critical point. So (i) is implied by Lemma 4.3. If $p \geq 50\left(d_{1}+1\right)$ then the lemma is implied by Lemma 5.3 and so we may assume $p<50\left(d_{1}+1\right)$. In particular, it follows that $r \leq d_{1}$ (if $r \geq 4$ then $r^{4}<p<50\left(d_{1}+1\right.$ ) implies $r<r \cdot r^{3} / 50<d_{1}+1$; if $r \leq 3$ then $p>200$ implies $d_{1}>3 \geq r$ ).

The proof of (ii) is by induction on $m$, but first we show that (i) and (ii) together imply (iii). If zero is the only critical point then (ii) immediately implies (iii) and so we assume henceforth that $r \geq 2$ and that $\nu(0)<d_{1}$.

If $m-t \leq 2 r$ then the upper bound in (iii) follows from the trivial bound $\left|S\left(f, p^{m}\right)\right| \leq p^{m}$. Next write $m-t=2 r+1+j$ with $j \geq 0$ and set

$$
\Delta=p^{(t+2 r) /\left(d_{1}+1\right)} p^{m\left(1-1 /\left(d_{1}+1\right)\right)},
$$

the desired bound. We have

$$
\left|S\left(f, p^{m}\right)\right| \leq\left|S_{0}\left(f, p^{m}\right)\right|+\sum_{\alpha \neq 0}\left|S_{\alpha}\left(f, p^{m}\right)\right|
$$


For the first term we have the trivial bound

$$
\left|S_{0}\left(f, p^{m}\right)\right| \leq p^{m-1}=p^{\left(j-d_{1}\right) /\left(d_{1}+1\right)} \Delta .
$$

Now there are at most $p-1$ nonzero critical points, each of multiplicity $\leq r_{1}-1 \leq r-1$, and so by (i),

$$
\begin{aligned}
& \sum_{\alpha \neq 0}\left|S_{\alpha}\left(f, p^{m}\right)\right| \leq p \cdot p^{t / r} p^{m(1-1 / r)} \\
& =p^{\left(j\left(r-d_{1}-1\right)-r d_{1}-d_{1}-1\right) /\left(\left(d_{1}+1\right) r\right)} \Delta=p^{\left(j-d_{1}\right) /\left(d_{1}+1\right)-(1+j) / r} \Delta .
\end{aligned}
$$

Combining (5.6) and (5.7) we have, for $j \leq d_{1} / 2$,

$$
\left|S\left(f, p^{m}\right)\right| \leq p^{-d_{1} /\left(2\left(d_{1}+1\right)\right)}\left(1+p^{-1 / r}\right) \Delta<2 p^{-1 / 4} \Delta<\Delta,
$$

and for $d_{1}>j>d_{1} / 2$,

$$
\left|S\left(f, p^{m}\right)\right| \leq\left(p^{-d_{1} /(2 r)}+1\right) p^{-1 /\left(d_{1}+1\right)} \Delta \leq\left(r^{-2 d_{1} / r}+1\right) r^{-4 /\left(d_{1}+1\right)} \Delta<\Delta .
$$

If $j \geq d_{1}$ then by the bound in (ii) (replacing $\nu$ with $d_{1}-1$ ) we obtain

$$
\left|S_{0}\left(f, p^{m}\right)\right| \leq p^{(-j-1) /\left(d_{1}\left(d_{1}+1\right)\right)} \Delta \leq p^{-1 / d_{1}} \Delta .
$$

For the remaining critical points we use the upper bound of (5.7) replacing $j$ with $d_{1}$. Thus

$$
\left|S\left(f, p^{m}\right)\right| \leq\left(p^{-1 / d_{1}}+p^{\left(-d_{1}-1\right) / r}\right) \Delta \leq\left(r^{-4 / d_{1}}+r^{-4\left(d_{1}+1\right) / r}\right) \Delta<\Delta .
$$

We return to the task of proving (ii) by induction on $m$. The bound follows trivially from $\left|S_{0}\left(f, p^{m}\right)\right| \leq p^{m-1}$ if $m \leq \nu+1+t+2 r$, and so we assume $m \geq \nu+2+t+2 r$. By (2.9) we have

$$
m-\sigma \geq \nu+2+t+2 r-(\nu+1+t-\tau)=1+2 r+\tau \geq \tau+2,
$$

and by the recursion formula of Lemma $2.2,\left|S_{0}\left(f, p^{m}\right)\right|=p^{\sigma-1}\left|S\left(g_{0}, p^{m-\sigma}\right)\right|$, where $g_{0}(x)=p^{-\sigma} f(p x)$. Since $g_{0}$ has the same degree monomials as $f$ we can apply the induction assumption to $g_{0}$ and obtain,

$$
\left|S_{0}\left(f, p^{m}\right)\right| \leq p^{\sigma-1} p^{(\tau+2 r) /\left(d_{2}+1\right)} p^{(m-\sigma)\left(1-1 /\left(d_{2}+1\right)\right)},
$$

where $d_{2}:=d_{p}\left(p^{-\tau} g_{0}\right) \leq d_{1}$. Now by $(2.11), d_{2} \leq \nu$ and so replacing $d_{2}$ by $\nu$ in the previous inequality and using the upper bound in (2.9) we deduce the inequality in (ii).

6. Dealing with the primes that divide $k_{i}-k_{j}$ for some $i \neq j$. If $p \mid\left(k_{j}-k_{i}\right)$ for some $k_{i}<k_{j} \leq d_{1}$ then there may be nonzero critical points of multiplicity exceeding $r$ and so we have to argue more carefully. Let $f(x)$ be a sparse polynomial as in (1.2) of degree $d$ and set $d_{1}=d_{p}\left(p^{-t} f^{\prime}(x)\right)$. For any pair $(i, j)$ with $1 \leq i<j \leq r$ let $p_{i j}$ be the maximal prime divisor of $k_{j}-k_{i}$ (taking $p_{i j}=1$ in case $k_{j}-k_{i}=1$ ) and put

$$
\mathcal{P}=\left\{p_{i j}: 1 \leq i<j \leq r\right\} \text {. }
$$


Assume now that $p>4 r, p \mid\left(k_{j}-k_{i}\right)$ for some $k_{i}<k_{j} \leq d_{1}$ but that $p \notin \mathcal{P}$. Let

$$
p_{l s}=\min \left\{p_{i j}: p \mid\left(k_{j}-k_{i}\right), k_{i}<k_{j} \leq d_{1}\right\},
$$

and define

$$
M:=r d_{1} / p_{l s}
$$

Then if $p^{e} \|\left(k_{j}-k_{i}\right)$ is the maximum power of $p$ dividing any of the differences $k_{j}-k_{i}$ that actually occur in the critical point congruence for $S\left(f, p^{m}\right)$, it follows from Lemma 4.1 that the multiplicity $\nu$ of any nonzero critical point satisfies

$$
\nu<r p^{e} \leq r\left(k_{j}-k_{i}\right) / p_{i j} \leq M .
$$

Let $S^{*}\left(f, p^{m}\right)$ denote the sum over a reduced residue system (modulo $\left.p^{m}\right)$ as in (1.7). For $j \geq 0$ define $\mu_{j}, t_{j}$ by

$$
p^{\mu_{j}}\left\|\left(a_{1} p^{j k_{1}}, \ldots, a_{r} p^{j k_{r}}\right), \quad p^{\mu_{j}+t_{j}}\right\|\left(a_{1} k_{1} p^{j k_{1}}, \ldots, a_{r} k_{r} p^{j k_{r}}\right) .
$$

Then we can write

$$
S\left(f, p^{m}\right)=\sum_{j=0}^{m} S^{*}\left(f\left(p^{j} x\right), p^{m-j}\right)=\sum_{j=0}^{m} p^{\mu_{j}-j} S_{j}^{*},
$$

where for $0 \leq j \leq m$,

$$
S_{j}^{*}=S^{*}\left(p^{-\mu_{j}} f\left(p^{j} x\right), p^{m-\mu_{j}}\right) .
$$

The critical point congruence associated with the sum $S_{j}^{*}$ is just

$$
g_{j}(x):=p^{-\mu_{j}-t_{j}}\left(a_{1} k_{1} p^{j k_{1}} x^{k_{1}-1}+\ldots+a_{r} k_{r} p^{j k_{r}} x^{k_{r}-1}\right) \equiv 0(\bmod p) .
$$

Viewing $g_{j}(x)$ as a polynomial over $\mathbb{F}_{p}$ we observe that for any $j<m$ the largest degree term of $g_{j+1}(x)$ is at most the smallest degree term of $g_{j}(x)$. Indeed, if $p^{t_{j}+\mu_{j}} \| a_{I} k_{I} p^{j k_{I}}$ then $p^{t_{j}+\mu_{j}+k_{I}} \| a_{I} k_{I} p^{(j+1) k_{I}}$ and $p^{t_{j}+\mu_{j}+k_{I}+1} \| a_{l} k_{l} p^{(j+1) k_{l}}$ for $l>I$. It follows that the degrees of the $g_{j}$ are nonincreasing (with $j$ ) and that at most $r$ of the $g_{j}(x)$ can have more than one nonzero term. The rest of the $g_{j}(x)$ are monomials and therefore the associated sums $S_{j}^{*}$ are zero, provided $m-\mu_{j} \geq 2$. Thus there are at most $r$ values of $j \leq m$ for which $m-\mu_{j} \geq 2$ and $S_{j}^{*}$ is nonzero. Moreover, for these nonzero sums the multiplicity of any nonzero critical point is bounded above by $M$.

Say $d_{1}=k_{I}-1$ for some $I$. Then since $p^{t} \| a_{I} k_{I}$ it is easily seen that for $0 \leq j \leq m$,

$$
\mu_{j}+t_{j} \leq t+j\left(d_{1}+1\right) .
$$

We split the sum in (6.4) into two parts according as $m-t_{j}-\mu_{j} \geq 8 M$ or not. If this inequality holds then since $S_{j}^{*}$ has at most $p$ critical points, 
each of multiplicity $\leq M$, it follows from Lemma 2.3 that $p^{\mu_{j}-j}\left|S_{j}^{*}\right| \leq p^{\mu_{j}-j} 4 p p^{t_{j} / M} p^{\left(m-\mu_{j}\right)(1-1 / M)}=\frac{4 p}{p^{\left(m-\mu_{j}-t_{j}\right) /(2 M)}} \frac{p^{m-j}}{p^{\left(m-\mu_{j}-t_{j}\right) /(2 M)}}$. Now $\left(m-\mu_{j}-t_{j}\right) /(2 M) \geq 4$. Also, since $p>2 r, 2 M<d_{1}$ and so by (6.6),

$$
\frac{m-\mu_{j}-t_{j}}{2 M} \geq \frac{m-t-j\left(d_{1}+1\right)}{d_{1}+1}=\frac{m-t}{d_{1}+1}-j .
$$

It follows that

$$
p^{\mu_{j}-1}\left|S_{j}^{*}\right| \leq \frac{4}{p^{3}} p^{t /\left(d_{1}+1\right)} p^{m\left(1-1 /\left(d_{1}+1\right)\right)} .
$$

We consider next the set of $j$ for which $m-t_{j}-\mu_{j}<8 M$ and let $j_{0}$ denote the least such $j$. Then

$$
\sum_{j \geq j_{0}} p^{\mu_{j}-j}\left|S_{j}^{*}\right| \leq p^{m-j_{0}}=p^{(m-t) /\left(d_{1}+1\right)-j_{0}} p^{t /\left(d_{1}+1\right)} p^{m\left(1-1 /\left(d_{1}+1\right)\right)} .
$$

Now

$$
(m-t)-j_{0}\left(d_{1}+1\right) \leq 8 M+t_{j_{0}}+\mu_{j_{0}}-t-j_{0}\left(d_{1}+1\right) \leq 8 M=8 r d_{1} / p_{l s},
$$

by (6.6). Thus

$$
\sum_{j \geq j_{0}} p^{\mu_{j}-j}\left|S_{j}^{*}\right| \leq p^{8 r / p_{l s}} p^{t /\left(d_{1}+1\right)} p^{m\left(1-1 /\left(d_{1}+1\right)\right)} .
$$

From (6.7) and (6.8) we conclude that

$$
\begin{aligned}
\left|S\left(f, p^{m}\right)\right| & \leq\left(4 r / p^{3}+p^{8 r / p_{l s}}\right) p^{t /\left(d_{1}+1\right)} p^{m\left(1-1 /\left(d_{1}+1\right)\right)} \\
& \leq p^{8 r / p_{l s}}\left(1+1 / p^{2}\right) p^{t /\left(d_{1}+1\right)} p^{m\left(1-1 /\left(d_{1}+1\right)\right)} .
\end{aligned}
$$

This establishes

Lemma 6.1. Suppose that $p \mid\left(k_{j}-k_{i}\right)$ for some $k_{i}<k_{j} \leq d_{1}, p>4 r$ and $p \notin \mathcal{P}$. Then

$$
\left|S\left(f, p^{m}\right)\right| \leq\left(1+1 / p^{2}\right) p^{8 r / p_{l s}} p^{t /\left(d_{1}+1\right)} p^{m\left(1-1 /\left(d_{1}+1\right)\right)}
$$

for some $p_{l s} \in \mathcal{P}$ with $p \mid\left(k_{l}-k_{s}\right), p<p_{l s}$.

7. Proof of Theorem 1.1. For any prime power $p^{m}$ and polynomial $f$ let

$$
R\left(f, p^{m}\right)=\frac{\left|S\left(f, p^{m}\right)\right|}{p^{m(1-1 / d)}} .
$$

Let $f$ be a sparse polynomial with $r$ terms and let $q$ be a positive integer such that $d_{p}(f) \geq 1$ for all prime divisors $p$ of $q$. Write

$$
\prod_{p^{m} \| q} R\left(f, p^{m}\right)=P_{1} P_{2} P_{3} P_{4} P_{5} P_{6}
$$


where the $P_{i}$ are products over the prime power divisors of $q$ satisfying the following constraints (counting prime powers only once if they happen to satisfy more than one constraint):

$$
\begin{aligned}
& P_{1}=\prod_{m=1} R\left(f, p^{m}\right), \\
& P_{2}=\prod_{\substack{1<m \leq t+1 \\
P_{3}}} R\left(f, p^{m}\right), \\
& P_{4}=\prod_{\substack{p \leq C_{3} \text { or } \\
p \leq r^{4} \text { or } p \in \mathcal{P}}} R\left(f, p^{m}\right), \\
& P_{5}=\prod_{\substack{p>r^{4} \\
p \mid\left(k_{j}-k_{i}\right) \text { for some } k_{i}<k_{j} \leq d_{1}, p \notin \mathcal{P}}} R\left(f, p^{m}\right), \\
& P_{6}=\prod_{\substack{\left.p \nmid t+2,50 d>p>r_{j}-k_{i}\right) \text { for all } k_{i}<k_{j} \leq d_{1} \text { and } \\
m \geq t+2, p>r^{4}, p>C_{2} \text { and } \\
p \geq 50 d}} R\left(f, p^{m}\right),
\end{aligned}
$$

where $C_{2}, C_{3}$ are the constants in Lemmas 5.3 and 5.4 respectively, and $\mathcal{P}$ is the set (6.1) of exceptional primes. By (1.6) the theorem follows if we show that each of the products $P_{i}$ is bounded by a constant depending only on $r$.

By Lemma 5.2, the Weil bound (5.1) and the trivial bound $R(f, p) \leq p^{1 / d}$ we have

$$
\begin{aligned}
P_{1} & \leq \prod_{p<C_{2}} R(f, p) \prod_{p \leq r^{4}} R(f, p) \prod_{p<50 d} R(f, p) \\
& \leq(1.75)^{C_{2}+r^{4}} \prod_{p<50 d} p^{1 / d} \ll(1.75)^{r^{4}} .
\end{aligned}
$$

For the next few products we need the following

Lemma 7.1. Let $f$ be a sparse polynomial with $r$ terms of degree $d$. For any prime $p$ let $t_{p}=\operatorname{ord}_{p}\left(f^{\prime}(x)\right)$. Then letting $p$ run through the set of all primes for which $d_{p}(f) \geq 1$ we have

$$
\prod_{p, d_{p}(f) \geq 1} p^{t_{p}} \leq d^{r} .
$$

Proof. Let $f(x)=a_{1} x^{k_{1}}+\ldots+a_{r} x^{k_{r}}$ and $p$ be a prime with $d_{p}(f) \geq 1$. Then for some $i, p \nmid a_{i}$, and so for this value of $i, p^{t_{p}} \mid k_{i}$. Thus the product over all such $p^{t_{p}}$ is a divisor of $k_{1} \ldots k_{r}$. 
(We continue to write $t$ for $t_{p}$.) For $P_{2}$ the condition $1<m \leq t+1$ implies that $t \geq 1$ and so $m \leq 2 t$. Thus we trivially have

$$
P_{2} \leq \prod_{p} p^{m / d} \leq \prod_{p} p^{2 t / d} \leq d^{2 r / d} \leq 2.1^{r} .
$$

The number of primes in the product $P_{3}$ is less than $r^{4} / 2+r^{2}+C_{3}<$ $r^{4}+C_{3}$ and so by Lemma 1.1, $P_{3} \leq 5^{r^{4}+C_{3}}$. For $P_{4}$ we apply Lemma 6.1 , to obtain

$$
\begin{aligned}
P_{4} & \leq \prod_{p}\left(1+\frac{1}{p^{2}}\right)\left(\prod_{p} p^{t / d}\right) \prod_{1 \leq i<j \leq r} \prod_{p \leq p_{i, j}} p^{8 r / p_{i j}} \\
& \ll d^{r / d} \prod_{1 \leq i<j \leq r} C_{5}^{4 r} \ll 1.5^{r} C_{5}^{2 r^{3}}
\end{aligned}
$$

for some absolute constant $C_{5}$. We may take $C_{5}=\sup _{x} e^{\theta(x) / x}$, where $\theta(x)=$ $\sum_{p \leq x} \log p$.

For $P_{5}$, we apply Lemma 5.4(iii) to obtain,

$$
P_{5} \leq \prod_{p<50 d} p^{(2 r+t) / d} \leq \prod_{p} p^{t / d} \prod_{p<50 d} p^{2 r / d} \leq d^{r / d} e^{2 r \theta(50 d) / d} \leq 1.5^{r} C_{5}^{100 r} .
$$

Finally, we apply Lemma 5.3 to $P_{6}$ to obtain

$$
P_{6} \leq \prod_{p} p^{t / d} \leq d^{r / d} \leq 1.5^{r} .
$$

Thus the product $P_{1} P_{2} P_{3} P_{4} P_{5} P_{6}$ is bounded above by a constant depending only on $r$.

\section{References}

[1] J. H. H. Chalk, On Hua's estimate for exponential sums, Mathematika 34 (1987), 115-123.

[2] J. R. Chen, On the representation of natural numbers as a sum of terms of the form $x(x+1) \ldots(x+k-1) / k$ !, Acta Math. Sinica 8 (1958), 253-257.

[3] -, On Professor Hua's estimate of exponential sums, Sci. Sinica 20 (1977), 711-719.

[4] T. Cochrane, Exponential sums modulo prime powers, Acta Arith. 101 (2002), 131149.

[5] T. Cochrane, C. Pinner and J. Rosenhouse, Bounds on exponential sums and the polynomial Waring's problem mod p, preprint.

[6] T. Cochrane and Z. Y. Zheng, Pure and mixed exponential sums, Acta Arith. 91 (1999), 249-278.

[7] -, - Exponential sums with rational function entries, ibid. 95 (2000), 67-95.

[8] —, - On upper bounds of Chalk and Hua for exponential sums, Proc. Amer. Math. Soc. 129 (2001), 2505-2516.

[9] P. Ding, An improvement to Chalk's estimation of exponential sums, Acta Arith. 59 (1991), 149-155.

[10] —, On a conjecture of Chalk, J. Number Theory 65 (1997), 116-129.

[11] L. K. Hua, On exponential sums, J. Chinese Math. Soc. 20 (1940), 301-312. 
[12] L. K. Hua, On exponential sums, Sci. Record (Peking) (N.S.) 1 (1957), 1-4.

[13] —, Additive Primzahltheorie, Teubner, Leipzig, 1959, 2-7.

[14] S. V. Konyagin and I. E. Shparlinski, Character Sums with Exponential Functions and Their Applications, Cambridge Univ. Press, Cambridge, 1999.

[15] W. K. A. Loh, On Hua's lemma, Bull. Austral. Math. Soc. 50 (1994), 451-458.

[16] - Exponential sums on reduced residue systems, Canad. Math. Bull. 41 (1998), 187-195.

[17] M. Lu, A note on the estimation of a complete rational trigonometric sum, Acta Math. Sinica 27 (1984), 817-823 (in Chinese).

[18] —, Estimate of a complete trigonometric sum, Sci. Sinica Ser. A 28 (1985), 561-578.

[19] - A note on complete trigonometric sums for prime powers, Sichuan Daxue Xuebao 26 (1989), 156-159.

[20] V. I. Nečaev [V. I. Nechaev], An estimate of the complete rational trigonometric sum, Mat. Zametki 17 (1975), 839-849 (in Russian); English transl.: Math. Notes 17 (1975), 504-511.

[21] - , On the least upper bound on the modulus of complete trigonometric sums of degrees three and four, in: Investigations in Number Theory, Saratov. Gos. Univ., Saratov, 1988, 71-76 (in Russian).

[22] V. I. Nečaev [V. I. Nechaev] and V. L. Topunov, Estimation of the modulus of complete rational trigonometric sums of degree three and four, Trudy Mat. Inst. Steklov. 158 (1981), 125-129 (in Russian); English transl. in: Analytic Number Theory, Mathematical Analysis and Their Applications, Proc. Steklov Inst. Math. 158 (1983), 135-140.

[23] M. Qi and P. Ding, Estimate of complete trigonometric sums, Kexue Tongbao 29 (1984), 1567-1569.

[24] - - - On an estimate of complete trigonometric sums, Chinese Ann. Math. Ser. B 6 (1985), 109-120.

[25] - - - Further estimates of complete trigonometric sums, J. Tsinghua Univ. Sci. Technol. 29 (1989), no. 6, 74-85.

[26] I. E. Shparlinski, On bounds of Gaussian sums, Mat. Zametki 50 (1991), 122-130 (in Russian).

[27] - On Gaussian sums for finite fields and elliptic curves, in: Algebraic Coding (First French-Soviet Workshop, Paris, 1991), Lecture Notes in Comput. Sci. 573, Springer, 1992, 5-15.

[28] —, On exponential sums with sparse polynomials and rational functions, J. Number Theory 60 (1996), 233-244.

[29] S. B. Stečkin [S. B. Stechkin], Estimate of a complete rational trigonometric sum, Trudy Mat. Inst. Stoklov. 143 (1977), 188-220 (in Russian); English transl.: Proc. Steklov. Inst. Math. 143 (1980), 201-220.

[30] A. Weil, On some exponential sums, Proc. Nat. Acad. Sci. U.S.A. 34 (1948), 204207.

[31] M. Zhang and Y. Hong, On the maximum modulus of complete trigonometric sums, Acta Math. Sinica (N.S.) 3 (1987), 341-350.

Department of Mathematics

Kansas State University

Manhattan, KS 66506, U.S.A.

E-mail: cochrane@math.ksu.edu pinner@math.ksu.edu jasonr@math.ksu.edu 